\title{
On a Method to Subtract off a Singularity at a Corner for the Dirichlet or Neumann Problem
}

\author{
By Neil M. Wigley*
}

\begin{abstract}
Let $D$ be a plane domain partly bounded by two line segments which meet at the origin and form there an interior angle $\pi \alpha>0$. Let $U(x, y)$ be a solution in $D$ of Poisson's equation such that either $U$ or $\partial U / \partial n$ (the normal derivative) takes prescribed values on the boundary segments. Let $U(x, y)$ be sufficiently smooth away from the corner and bounded at the corner. Then for each positive integer $N$ there exists a function $V_{N}(x, y)$ which satisfies a related Poisson equation and which satisfies related boundary conditions such that $U-V_{N}$ is $N$-times continuously differentiable at the corner. If $1 / \alpha$ is an integer $V_{N}$ may be found explicitly in terms of the data of the problem for $U$.
\end{abstract}

In solving an elliptic partial differential equation by numerical methods the results proved about convergence of the numerical approximation to the actual solution frequently depend on differentiability properties of the (unknown) solution. In the work of Gerschgorin [2] and other papers written since, it is assumed that the solution of the partial differential equation has derivatives of order four which are continuous up to the boundary. If the boundary and all the data are sufficiently smooth there is, of course, no problem. In many cases, however, the boundary possesses a finite number of singularities, usually (in the two-dimensional case) in the form of corners; occasionally too, the boundary data may have jumps. Laasonen [3] has proved that convergence of the discrete solution to the actual solution holds for the Dirichlet problem, but that the convergence is slow in a neighborhood of the corner.

In this paper we will consider a method to subtract off the singularity. The method is quite old (see Fox [1]), but includes results on the asymptotic behavior of solutions near a corner. In this light see the works of Lewy [4], Lehman [5], Wasow [6], and the author [7]. We consider a problem for which the solution is not known to be smooth. We then find, explicitly in terms of the boundary data, a solution to a related problem; then the difference between these two solutions is a solution to a third problem, and is sufficiently well-behaved to insure convergence of difference schemes. Finally, the sought solution can be found by adding the explicitly given one to the numerically-solved one.

Let $D$ be a plane domain partly bounded by two open line segments $\Gamma_{1}$ and $\Gamma_{2}$, which share the origin as a common endpoint and form there an interior angle $\pi \alpha>0$. We assume that $\Gamma_{1}$ is a subset of the positive $x$-axis and $\Gamma_{2}$ makes an angle $\pi \alpha>0$ with the positive $x$-axis. Let $F(x, y)$ be given in $D$ and $\Phi_{i}(x, y)$ (respectively $\left.\Psi_{i}(x, y)\right)$ be given on $\Gamma_{i}(i=1,2)$. Let $U(x, y)$ be a solution of $\Delta U=F$ in $D$, and have boundary values $U(x, y)=\Phi_{i}(x, y)$ on $\Gamma_{i}$ (respectively $\partial U / \partial n=\Psi_{i} ; \partial / \partial n$ is

Received January 16, 1967, revised July 18, 1968.

* The author is indebted to the referee for many suggestions used in the preparation of this. paper. 
the outward normal derivative). We assume that $U$ is bounded and has continuous second derivatives in $D$, and is continuous (respectively has continuous first derivatives) in $D \cup \Gamma_{1} \cup \Gamma_{2}$. Throughout the paper we have the independent variables $z=x+i y=r e^{i \theta}$. Depending on circumstances we will sometimes write $u(z, \bar{z})$ for $u(x, y)$.

In general $U$ is smooth along $\Gamma_{1}$ and $\Gamma_{2}$ but possesses singularities of its derivatives at the corner. We do, however, have knowledge of the asymptotic behavior of $U$ and its derivatives (see [5], [6], [7]). For simplicity let us assume that $F$ is analytic in $D \cup \Gamma_{1} \cup \Gamma_{2} \cup\{0\}$ and that $\Phi_{i}$ (respectively $\Psi_{i}$ ) is analytic on $\bar{\Gamma}_{i}$. Weaker conditions on the data could be used [7]. Let $N$ be an integer $\geqq 2$. Then there exists a polynomial $P_{N}\left(z, \bar{z}, z^{1 / \alpha}, \bar{z}^{1 / \alpha}, \log z, \log \bar{z}\right)$ such that as $z \rightarrow 0, z \in D \cup \Gamma_{1} \cup \Gamma_{2}$,

$$
U(x, y)=P_{N}\left(z, \bar{z}, z^{1 / \alpha}, \bar{z}^{1 / \alpha}, \log z, \log \bar{z}\right)+o\left(z^{N}\right) .
$$

Derivatives of $U(x, y)$ of order $\leqq N$ have asymptotic expansions obtained by differentiating (1) formally; thus the $k$ th derivative of the error term $o\left(z^{N}\right)$ is an error term $o\left(z^{N-k}\right)$. If we wish to examine the derivatives of $U(z)$ of order $\leqq 4$ we can take $N=4$. Then the error term will have continuous fourth derivatives, and the singularities of $U(z)$ and its derivatives of order $\leqq 4$ are exhibited in the polynomial $P_{N}$. Thus for numerical purposes it would be desirable to calculate, in terms of the data, the coefficients of $P_{N}$; or at least the coefficients of those monomials of $P_{N}$ which do not have fourth order derivatives at $z=0$.

In this paper we will indicate a method to find most of the coefficients. To be more specific we will give an algorithm to calculate all of the coefficients of $P_{N}$ except those which are coefficients of monomials of the form $\left(z^{m / \alpha}-\bar{z}^{m / \alpha}\right)$. Thus the singularities of $U$ of order $\leqq N$ are known explicitly up to some polynomial

$$
Q_{N}=\sum_{m=0}^{[N \alpha]} A_{m}\left(z^{m / \alpha}-\bar{z}^{m / \alpha}\right)
$$

where [ ] is the greatest integer.

Let us assume for the moment that the algorithm has been demonstrated. Then we have two cases.

Case I. $1 / \alpha$ is an integer. Then $Q_{N}$ is smooth at the origin, and the singularities of $U(x, y)$ of order $\leqq N$ are known explicitly. Let us use the symbol $R_{N}$ to denote the polynomial formed by taking those monomials of $P_{N}$ which have singular derivatives; since $1 / \alpha$ is an integer, these will be precisely the terms of $P_{N}$ which involve logarithms. Then the function $V=U-R_{N}$ belongs to $C^{N}$ at the origin. If we then replace the original differential equation $\Delta U=F, U=\Phi_{i}\left(\partial U / \partial n=\Psi_{i}\right)$ with the problem $\Delta V=F-\Delta R_{N}, V=\Phi_{i}-R_{N}\left(\partial V / \partial n=\Psi_{i}-\partial R_{N} / \partial n\right)$, we know in advance that the solution $V=V_{N}$ will belong to $C^{N}$. Thus we can solve the new problem numerically and are assured of convergence of the discrete solution to the actual solution. Then by adding $R_{N}$ to this discrete solution of the new problem we get a good approximation to $U(x, y)$.

Case II. $1 / \alpha$ is not an integer (the most common case in practice seems to be $\alpha=3 / 2$ ). If the proof of Gerschgorin's results are examined (see, e.g., Forsythe and Wasow [8, pp. 283-288]) it is seen that the difficulty lies with the unboundedness near $z=0$ of certain fourth derivatives of $U(x, y)$. Let us, as before, construct the polynomial $R_{N}$ consisting of the singular terms of $P_{N}$. Then the function $V_{N}$ has for 
its singularities precisely those monomials of $Q_{N}=\sum A_{m}\left(z^{m / \alpha}-\bar{z}^{m / \alpha}\right)$. It may be possible to devise a numerical method of approximating the coefficients $A_{m}$. Suppose that $\widetilde{A}_{m}$ is such an approximation and that $\widetilde{V}_{N}$ is the function obtained by replacing $Q_{N}$ with $\widetilde{Q}_{N}=\sum \tilde{A}_{m}\left(z^{m / \alpha}-\bar{z}^{m / \alpha}\right)$. Then $\tilde{V}_{N}$ will have singular derivatives, but if the approximation $\widetilde{A}_{m}$ of $A_{m}$ is good enough the singularities may still be small. Thus for a given mesh size $h$ one could approximate $A_{m}$ with $\tilde{A}_{m}(h)$, then find a discrete approximation $\tilde{V}(h)$ to $\tilde{V}$ using the Gerschgorin method. Knowing $\tilde{V}(h)$ one could then find a better approximation $\widetilde{A}_{m}(h / 2)$ to $A_{m}$, and then repeat the process.

We now discuss the algorithm for finding the coefficients. First we reduce the problem to the simpler case $F \equiv 0$. Consider the transformation $\zeta=z^{1 / \alpha}$. In a neighborhood of the corner it has the effect of straightening out the corner; in fact it maps a neighborhood of the corner conformally into some semicircle about the origin in the upper half of the $\zeta$-plane and maps $\Gamma_{1}$ and $\Gamma_{2}$ into the diameter of the semicircle. We define $u(\zeta)$ by $u(\zeta)=U(z)$ and then, for some $r_{0}>0$, we have

$$
\Delta u(z)=\alpha^{2} \sum_{p, q=0}^{\infty} a_{p q} z^{(p+1) \alpha-1} \bar{z}^{(q+1) \alpha-1}
$$

for $0<|z|<r_{0}$, Im $z>0$. Here the $a_{p q}$ come from the complex form

$$
F(x, y)=\sum_{p, q=0}^{\infty} a_{p q} z^{p} \bar{z}^{q}
$$

of the Taylor series of $F$.

Since

$$
\Delta u=4 \frac{\partial^{2}}{\partial z \partial \bar{z}} u
$$

where $\partial / \partial z=\frac{1}{2}(\partial / \partial x-i \partial / \partial y), \partial / \partial \bar{z}=\frac{1}{2}(\partial / \partial x+i \partial / \partial y)$, we see by integration with respect to $z$ and $\bar{z}$ that if $u$ were asymptotic to a power series in $z^{\alpha}$ and $\bar{z}^{\alpha}$, then $u$ would be asymptotic to the series

$$
u(x, y)=\frac{\alpha^{2}}{4} \sum_{p, q=0}^{\infty} \frac{1}{(p+1) \alpha(q+1) \alpha} a_{p q} z^{(p+1) \alpha} \bar{z}^{(q+1) \alpha} .
$$

If we define $v(z, \bar{z})$ to be the right side of (3), then the function $w=u-v$ satisfies $\Delta w=0$. Setting $V(z, \bar{z})=v\left(z^{1 / \alpha}, \bar{z}^{1 / \alpha}\right)$ and $W(z, \bar{z})=w\left(z^{1 / \alpha}, \bar{z}^{1 / \alpha}\right)$ we have $W=U-$ $V$ where

$$
V(z, \bar{z})=\frac{1}{4} \sum_{p, q=0}^{\infty} \frac{a_{p q}}{(p+1)(q+1)} z^{p+1} \bar{z}^{q+1} .
$$

Thus if we set $\tilde{\Phi}_{i}=\Phi_{i}-V$ (respectively $\Psi_{i}=\Psi_{i}-\partial V / \partial n$ ), we have $\Delta W=0$ in $D, W=\tilde{\Phi}_{i}\left(\partial W / \partial n=\tilde{\Psi}_{i}\right)$ on $\Gamma_{i}$.

Thus by replacing $U$ with $W$ and $\Phi_{i}$ with $\tilde{\Phi}_{i},\left(\Psi_{i}\right.$ with $\left.\tilde{\Psi}_{i}\right)$ we can assume $F \equiv 0$ and $\Delta U=0$.

Now let us assume that $U$ is prescribed on $\Gamma_{1} \cup \Gamma_{2}$. Thus we have $\Delta U=0$ in $D$ and $U=\Phi_{i}$ on $\Gamma_{i}$. Let the Taylor series for $\Phi_{1}$ and $\Phi_{2}$ be

$$
\Phi_{1}(r)=\sum_{\nu=0}^{\infty} a_{\nu} r^{\nu} \quad \text { and } \quad \Phi_{2}(r)=\sum_{\nu=0}^{\infty} b_{\nu} r^{\nu}
$$


where $r=|z|$ denotes arc length on $\Gamma_{i}$. For every integer $N \geqq 2$ we will find a polynomial $v_{N}\left(z^{\alpha}, \bar{z}^{\alpha}, \log z, \log \bar{z}\right)$ such that $w_{N}=u-v_{N}$ satisfies $\Delta w_{N}=0$ in $D$ and $w_{N}(x, 0)=o\left(|x|^{N}\right)$ on $\operatorname{Im} z=0$.

For convenience of notation we drop the subscript $N$.

We begin by setting

$$
v_{1}(z, \bar{z})=\frac{1}{2} \sum_{\nu=0}^{\infty} a_{\nu}\left(z^{\nu \alpha}+\bar{z}^{\nu \alpha}\right) .
$$

Then for $z=\bar{z}=r>0$ we have

$$
v_{1}(r, r)=\sum_{\nu=0}^{\infty} a_{\nu} r^{\nu \alpha}=u(r, r),
$$

and for $z=\bar{z}=-r<0$, if $w_{1}=u-v_{1}$,

$$
w_{1}(z, \bar{z})=\sum_{\nu=0}^{\infty} b_{\nu} r^{\nu \alpha}-\sum_{\nu=0}^{\infty} a_{\nu}(\cos \nu \pi \alpha) r^{\nu \alpha} .
$$

We now look for a function $v_{2}(z, \bar{z})$ which is harmonic, vanishes on the positive $x$-axis in a neighborhood of the origin and agrees with $w_{1}(z, \bar{z})$ on the negative $x$-axis in a neighborhood of the origin. It is reasonable to hope that such a function would be given by powers of $z^{\nu \alpha}-\bar{z}^{\nu \alpha}$. Indeed, for irrational $\alpha$ we can choose $v_{2}$ by

$$
v_{2}=\sum_{\nu=0}^{\infty} \frac{b_{\nu}-a_{\nu} \cos \nu \pi \alpha}{2 i \sin \nu \pi \alpha}\left(z^{\nu \alpha}-\bar{z}^{\nu \alpha}\right) .
$$

It may happen, of course, that this series does not converge; in this case we take, for $N \geqq 2$,

$$
v_{2}=\sum_{\nu=0}^{N} \frac{b_{\nu}-a_{\nu} \cos \nu \pi \alpha}{2 i \sin \nu \pi \alpha}\left(z^{\nu \alpha}-\bar{z}^{\nu \alpha}\right) .
$$

Then with $w(z, \bar{z})=w_{1}(z, \bar{z})-v_{2}(z, \bar{z})=u(z, \bar{z})-v_{1}(z, \bar{z})-v_{2}(z, \bar{z})$ we get

$$
\begin{aligned}
\Delta w(z, \bar{z}) & =0 \quad \text { for } 0 \leqq|z|<r_{0}, \\
w(x, x) & =0 \text { for } x>0, \\
w(x, x) & =o\left(|x|^{N \alpha}\right) \quad \text { for } x<0 .
\end{aligned}
$$

If $\alpha$ is rational, the preceding method fails precisely for those terms of $v_{2}(z, \bar{z})$ for which $\sin \nu \pi \alpha=0$. We now indicate how to modify $v_{2}(z, \bar{z})$ in this case. Let $\alpha=p / q$, $\operatorname{gcd}(p, q)=1$, and let us concentrate first on the problem of finding a function $v_{2}=(z, \bar{z})$ which is harmonic, vanishes on the positive real axis, and on the negative real axis satisfies, for some integer $k \geqq 0$,

$$
v_{2}(x, x)=\left(b_{k q}-a_{k q} \cos k q \pi \alpha\right) r^{k q}=\left(b_{k q}-a_{k q} \cos k p \pi\right) r^{k p} .
$$

It happens that the introduction of terms in $\log z$ and $\log \bar{z}$ will give such a function $v_{2}(z, \bar{z})$. In fact, we take

$$
v_{2}(z, \bar{z})=\frac{(-1)^{k p}}{2 \pi i}\left(b_{k q}-a_{k q} \cos k p \pi\right)\left(z^{k p} \log z-\bar{z}^{k p} \log \bar{z}\right) .
$$

For the general case, where $k$ now varies over the positive integers, we take 


$$
\begin{aligned}
v_{2}(z, \bar{z})= & \sum_{\nu=0: \nu \neq 0(\bmod q)}^{N} \frac{b_{\nu}-a_{\nu} \cos \nu \pi \alpha}{2 i \sin \nu \pi \alpha}\left(z^{\nu}-\bar{z}^{\nu}\right) \\
& +\sum_{k=0}^{[N / p]} \frac{(-1)^{k p}}{2 \pi i}\left(b_{k q}-a_{k q} \cos k p \pi\right)\left(z^{k p} \log z-\bar{z}^{k p} \log \bar{z}\right) .
\end{aligned}
$$

Then as before $w(z, \bar{z})=u(z, \bar{z})-v_{1}(z, \bar{z})-v_{2}(z, \bar{z})$ satisfies

$$
\begin{aligned}
\Delta w(z, \bar{z}) & =0 \quad \text { for } 0 \leqq|z|<r_{0}, \\
w(x, x) & =0 \text { for } x>0, \\
w(x, x) & =o\left(|x|^{N \alpha}\right) \quad \text { for } x<0 .
\end{aligned}
$$

In both cases we have constructed a function $v(z, \bar{z})$ such that $w(z, \bar{z})=u(z, \bar{z})-$ $v(z, \bar{z})$ has derivatives at the origin of order $\leqq[N \alpha]$. Thus $w(z, \bar{z})$ has a Taylor expansion at the origin and we can write

$$
u(z, \bar{z})=\sum_{m, n=0}^{[N \alpha]-1} 1_{m, u} z^{m} \bar{z}^{n}+o\left(|z|^{[N \alpha]}\right) .
$$

Since $w(z, \bar{z})$ is harmonic, real-valued, and vanishes on the positive $x$-axis, we can rewrite (7) in the form

$$
w(z, \bar{z})=\sum_{n=0}^{[N \alpha]-1} A_{n}\left(z^{n}-\bar{z}^{n}\right)+o\left(|z|^{[N \alpha]}\right)
$$

where $A_{n}=A_{n 0}-A_{0 n}$ and all the other $A_{m n}$ 's vanish. There is no other information about the $A_{n}$ 's which is obtainable without turning to global considerations of the original differential equation and its domain. Indeed, if $A_{n}{ }^{\prime}$ are arbitrary pure imaginary numbers then

$$
\hat{w}(z, \bar{z})=\sum_{n=0}^{[N \alpha]-1} A_{n}{ }^{\prime}\left(z^{n}-\bar{z}^{n}\right)
$$

satisfies all the properties listed for $w(z, \bar{z})$.

We now set $W(z, \bar{z})=w\left(z^{1 / \alpha}, \bar{z}^{1 / \alpha}\right)$ and $V(z, \bar{z})=v\left(z^{1 / \alpha}, \bar{z}^{1 / \alpha}\right)$, and get

$$
W(z, \bar{z})=\sum_{n=0}^{[N \alpha]-1} A_{n}\left(z^{n / \alpha}-\bar{z}^{n / \alpha}\right)+o\left(|z|^{[N \alpha] / \alpha}\right)
$$

where $W(z, \bar{z})=U(z, \bar{z})-V(z, \bar{z})$. The function $W(z, \bar{z})$ is the function $Q_{N}$ mentioned in the introduction, and $V(z, \bar{z})+W(z, \bar{z})$ is the function $P_{N}$.

For the case of the Neumann boundary condition for $u(z, \bar{z})$ we proceed in exactly the same way. Since the calculations are somewhat more tedious but intrinsically no more difficult, we merely state the results. In particular let us restrict ourselves to the case $\alpha=1 / n, n$ an integer. Let $\partial U / \partial n=\Psi_{1}=\sum_{\nu=0}^{\infty} a_{\nu} r^{r}$ on $\Gamma_{1}$, and $\partial U / \partial n=\Psi_{2}=\sum_{\nu=0}^{\infty} b_{v} r^{\nu}$ on $\Gamma_{2}$. If $n$ is odd we set

$$
\begin{aligned}
v(z, \bar{z})= & \sum_{\nu=1 ; \nu \neq 0(\bmod n)}^{N-1} \frac{1}{2 \nu S_{\nu}}\left(b_{\nu-1}-a_{\nu-1} C_{\nu}\right)\left(z^{\nu}+\bar{z}^{\nu}\right)+\sum_{\nu=1}^{N-1} \frac{i a_{\nu-1}}{2 \nu}\left(z^{\nu}-\bar{z}^{\nu}\right) \\
& +\sum_{k=1}^{[N / n]} n A_{k}\left(z^{n k} \log z+\bar{z}^{n k} \log \bar{z}\right)
\end{aligned}
$$

where 


$$
\begin{aligned}
S_{\nu} & =\sin (\nu / n-1) \pi, \quad C_{\nu}=\cos (\nu / n-1) \pi \\
2 \pi n k A_{k} & =(-1)^{k-1}\left(b_{n k-1}+(-1)^{k} a_{n k-1}\right) .
\end{aligned}
$$

If $n$ is even, $n=2 m$, set

$$
\begin{aligned}
V(z, \bar{z})= & \sum_{\nu \neq 0, m(\bmod n) ; 0 \leqq \nu \leqq N-1} \frac{b_{\nu-1}-a_{\nu-1} C_{\nu}}{2 \nu S_{\nu}}\left(z^{\nu}+\bar{z}^{\nu}\right) \\
& +\sum_{\nu \equiv m(\bmod } \sum_{n) ; m \leqq \nu \leqq N-1} \frac{b_{\nu-1}}{2 \nu S_{\nu}}\left(z^{\nu}+\bar{z}^{\nu}\right)-\sum_{k=1}^{[N / n]} \frac{a_{n k-1}+(-1)^{k} b_{n k-1}}{2 \pi k} \\
& \times\left(z^{n k} \log z+\bar{z}^{n k} \log \bar{z}\right)-\sum_{\nu=1}^{N-1} \frac{a_{-1}}{2 i \nu}\left(z^{\nu}-\bar{z}^{\nu}\right) .
\end{aligned}
$$

Then there are constants $A_{m}$ such that the function $W=U-V$ satisfies

$$
W(z, \bar{z})=\sum_{m=0}^{[N-1 / n]} A_{m}\left(z^{m n}+\bar{z}^{m n}\right)+o\left(z^{N}\right) .
$$

Example. Let $\alpha=3 / 2, p=3, q=2, F \equiv 0, \Phi_{1}=\alpha r^{2}+\beta r^{4}, \Phi_{2}=\gamma r^{2}+\delta r^{4}$. Then $a_{2}=\alpha, a_{4}=\beta, b_{2}=\gamma, b_{4}=\delta$.

Set

and

$$
v_{1}(z, \bar{z})=\frac{1}{2}\left[\alpha\left(z^{3}+\bar{z}^{3}\right)+\beta\left(z^{6}+\bar{z}^{6}\right)\right]
$$

$$
\begin{aligned}
V_{1}(z, \bar{z})= & \frac{1}{2}\left[\alpha\left(z^{2}+\bar{z}^{2}\right)+\beta\left(z^{4}+\bar{z}^{4}\right)\right] \\
v_{2}(z, \bar{z})= & -\frac{1}{2 \pi i}(\gamma-\alpha \cos 3 \pi)\left(z^{3} \log z-\bar{z}^{3} \log \bar{z}\right) \\
& +\frac{1}{2 \pi i}(\delta-\beta)\left(z^{6} \log z-\bar{z}^{6} \log \bar{z}\right)
\end{aligned}
$$

and

$$
\begin{aligned}
& V_{2}(z, \bar{z})=v_{2}\left(z^{\alpha}, \bar{z}^{\alpha}\right)=v_{2}\left(z^{q / p}, \bar{z}^{q / p}\right)=v_{2}\left(z^{2 / 3}, \bar{z}^{2 / 3}\right) \\
& V_{2}(z, \bar{z})=-\frac{\gamma+\alpha}{2 \pi i} \frac{2}{3}\left(z^{2} \log z-\bar{z}^{2} \log \bar{z}\right)+\frac{\delta-\beta}{2 \pi i} \frac{2}{3}\left(z^{4} \log z-\bar{z}^{4} \log \bar{z}\right) .
\end{aligned}
$$

Then $W(z, \bar{z})=U-V_{1}-V_{2}$ satisfies

$$
\begin{aligned}
W(z, \bar{z})=0 & \text { on } \Gamma_{1} \cup \Gamma_{2} \\
\Delta W=0 & \text { in } D .
\end{aligned}
$$

Thus $w(z, \bar{z})=W\left(z^{2 / 3}, \bar{z}^{2 / 3}\right)$ satisfies

$$
\Delta w=0 \text { for } 0<|z|<r_{0}, \lim z>0
$$

and

$$
w=0 \text { for } \lim z=0,-r_{0}<x<r_{0} .
$$

So $w(z)$ is analytic on $0 \leqq|z|<r_{0}$, and

$$
w(z, \bar{z})=\sum_{m=0}^{\infty} A_{m}\left(z^{m}-\bar{z}^{m}\right) .
$$


Then $W(z, \bar{z})=w\left(z^{3 / 2}, \bar{z}^{3 / 2}\right)=\sum_{m=0}^{\infty} A_{m}\left(z^{3 m / 2}-\bar{z}^{3 m / 2}\right)$, so $W$ differs from a $C^{4}$ function by the polynomial $Q(z, \bar{z})=A_{1}\left(z^{3 / 2}-\bar{z}^{3 / 2}\right)$. By picking a mesh size $h$ and getting an approximation to $W$, one may be able to get an approximation $A_{1}{ }^{1}$ to $A_{1}$. Then, with a mesh size $h_{1}<h$, one could get an approximation to $W_{1}=W-A_{1}^{1}\left(z^{3 / 2}-\bar{z}^{3 / 2}\right)$, and then get an approximation $A_{1}{ }^{2}$ to $A_{1}{ }^{1}$. It is not known whether this process works, but in some cases, where great accuracy is needed near the corner, it may be worth attempting.

University of North Carolina

Chapel Hill, North Carolina 27514

1. L. Fox, (Editor), Numerical Solution of Ordinary and Partial Differential Equations, Pergamon Press, London and Addison-Wesley, Reading, Mass., 1962, pp. 302-303. MR 26 \#4488.

2. S. GERSCHGORIN, "Fehlerabschätzung für das Differenzenverfahren für Lösung partieller Differentialgleichungen," Z. Angew. Math. Mech., v. 10, 1930, pp. 373-382.

3. P. LAASONEN, "On the behavior of the solution of the Dirichlet problem at analytic corners," Ann. Acad. Sci. Fenn. A I, v. 408, 1957, pp. 3-16.

4. H. LEWY, "Developments at the confluence of analytic boundary conditions," Univ. of Calif. Publ. Math., v. 1, 1950, pp. 247-280.

5. R. S. LEHMAN, "Developments at an analytic corner of solutions of elliptic partial differential equations," J. Math. Mech., v. 8, 1959, pp. 727-760. MR 21 \#4291.

6. W. R. WASOw, "Asymptotic development of the solution of Dirichlet's problem at analytic corners," Duke Math. J., v. 24, 1957, pp. 47-56. MR 18, 568 .

7. N. M. WIGLEY, "Asymptotic expansions at a corner of solutions of mixed boundary value problems," J. Math. Mech., v. 13, 1964, pp. 549-576. MR 29 \#2516.

8. G. E. Forsythe \& W. R. WAsow, Finite-Difference Methods for Partial Differential Equations, Appl. Math. Series, Wiley, New York, 1960, pp. 283-288. MR 23 B3156. 